\title{
Incidence of Predisposing Factors on the Human Hand-arm Response with Flexed and Extended Elbow Positions of Workers Subject to Different Sources of Vibrations
}

\author{
Massimo Cavacece* \\ University of Cassino and Southern Lazio - Via G. Di Biasio, 43 Cassino (FR) Italy
}

\begin{abstract}
Received September 28, 2021; Revised November 24, 2021; Accepted December 13, 2021
Cite This Paper in the following Citation Styles

(a): [1] Massimo Cavacece, "Incidence of Predisposing Factors on the Human Hand-arm Response with Flexed and Extended Elbow Positions of Workers Subject to Different Sources of Vibrations," Universal Journal of Public Health, Vol.9, No.6, pp. 507-519, 2021. DOI: 10.13189/ujph.2021.090620

(b): Massimo Cavacece, (2021). Incidence of Predisposing Factors on the Human Hand-arm Response with Flexed and Extended Elbow Positions of Workers Subject to Different Sources of Vibrations. Universal Journal of Public Health, 9(6), 507-519. DOI: 10.13189/ujph.2021.090620
\end{abstract}

Copyright $\odot 2021$ by authors, all rights reserved. Authors agree that this article remains permanently open access under the terms of the Creative Commons Attribution License 4.0 International License

\begin{abstract}
Purpose This paper analyses the predisposing factors that affect the time response of the hand-arm system. Predisposing factors are the method of tool use, tool type, source of power, the mass of tool, workpiece held in hand, dimensions of the handle, grip force and push force, and dynamic and thermal properties of handgrips.

exposures are considered to estimate the response of human hand-arm positions in the time domain.

Conclusions Mathematical model and experimental investigations provide a better evaluation of health risks and muscle actions associated with exposure to hand-transmitted vibration from power tools in work conditions.
\end{abstract}

Methods This research considers the variability of the time response of the hand-arm system with respect to predisposing factors evaluated in 5 working conditions. The mathematical model consists of a distributed parameter representation in the form of a beam characterized by a continuous distribution of the mass, damping, and elasticity of the physical properties of the human and-arm system. The mathematical model considers the action of the extensor muscles of the elbow. The experimental investigations evaluate the forced vibrations of the human hand-arm system in work conditions.

Novelty in research The analysis of predisposing factors on the human hand-arm response with flexed and extended elbow positions of workers subject to different sources of vibrations represents the novelty in this research. Operators assume different human hand-arm postures when using manual power tools. The human hand-arm with flexed and extended elbow positions are the postures of workers evaluated in this research. The research on postures of workers aims to provide an evidence base for prevention. Another important objective is to provide a better overview of the extent of the occupational burden of workers. Work-related diseases include musculoskeletal disorders.

Results Posture factors of the human hand-arm system and predisposing of occupational hand-transmitted vibration
Keywords Musculoskeletal Disorders Human Hand Forces Human Hand-Arm Postures Health \& Safety Workplace Human Factors Ergonomics

\section{Introduction}

The grip, push forces and working postures may be highly variable by using power tools in the working conditions. The forced vibrations on the hand-arm system can be generated in a wide range of frequency [1].

The probability and severity of the injury, caused by handtransmitted vibration, depend on causal predisposing factors and human arm-hand postures [2]. Causal factors can be the magnitude, frequency, direction, input positions, and duration of vibration. Predisposing factors are the method of tool use, tool type, source of power, the mass of tool, workpiece held in hand, dimensions of the handle, grip force and push force, and dynamic and thermal properties of handgrips. Operators assume different human hand-arm postures when using manual power tools [3]. 
The postures of the human hand-arm system and the variations in the forces of the hand on the tool handle cause different biodynamic responses of the human hand-arm system [4]. The postures of the hand-arm system are examined as a function of elbow flexion. Causal factors, predisposing factors, and the action of the extensor muscles of the elbow generate a complex interrelation that depends on the time of exposure [5].

As before mentioned, the time response of the hand-arm system for predisposing factors is investigated in 5 working conditions. The features of vibration acceleration signals are Peak, Peak-to-Peak, Max, RMS values [6]. The mathematical model consists of a distributed parameter representation in the form of a continuous distribution of the mass, damping, and elasticity of the physical properties of the human and-arm system. Considering the forced vibrations of the human hand-arm system in work conditions, the prediction examines the role of the extensor muscles of the elbow [7].

\section{Model of human hand-arm system}

An impact at the handle of an instrument provokes the following effects: it enters the hand, causes a shock wave, crosses the wrist along with the bones at the bottom of the arm, reaches the elbow. There are two types of mechanical waves: longitudinal and transverse. If the wave displacement vector, which represents the perturbation, is perpendicular to the propagation direction, the wave is transverse. If the displacement occurs in the same direction of propagation, the wave is longitudinal. The impact at the handle of the instrument generates longitudinal compression waves and reflected waves [8]. The shock wave propagates like a compression wave from the wrist to the elbow. If the wave reaches the elbow, there is a reflected wave. From the elbow, the reflected wave propagates to the wrist. The reflected wave propagates like a compression wave. The reflected wave depends on the reflection impedance in the elbow joint. The magnitude of the shock wave and the reflected wave depends on the angle of the elbow opening. The shock wave and the return wave generate traction and compression on the forearm, respectively. Traction is more damaging than bone compression. Tensile strength is about $75 \%$ of the compressive strength. Therefore, the effects of waves provoke discomfort on the elbow, the wrist, the elbow, and the forearm. These effects, generated by the impact on the instrument handle, can cause injuries to the joints of the elbow and wrist (Figures 1, 2 and 3).

The values of deformation in compression are greater than the values of the tensile deformation of the bones [9]. The difference in mechanical properties in traction and compression is caused by the uneven anisotropy of the bone structure. The tensile modulus of elasticity is different from the compressive modulus of elasticity. If the bone is tested under various loading conditions, anisotropy causes several dynamic responses of the bone. Therefore, the maximum bending resistance of the bone is different from the maximum shear resistance under torsional load conditions. Similarly, the torsion modulus of elasticity differs from the bending modulus. The mechanical



Figure 1. Extended human hand-arm system

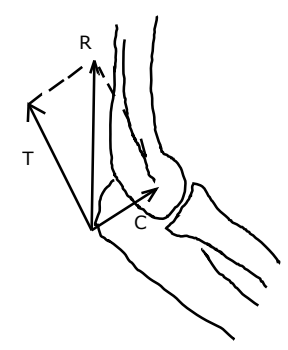

Figure 2. Obtuse angle of elbow



Figure 3. Acute angle of elbow 
characteristics of bone depend on age and stress conditions.

The tension developed by the muscle during contraction depends on the length of the muscle fibers at the initial moment of muscle contraction. Muscle tension presents the following two components: passive tension and active tension. Passive tension varies with muscle elongation. Passive tension depends on the length of the muscle, the elastic components of the muscle, the connective tissue. Active tension varies with muscle contraction. Total tension is the sum of passive tension and active tension. The initial muscle length generates different values of passive tension. The initial length activates the muscle tension-length curve. The contributions of active and passive tension participate in a normal working condition. The values of the elastic constants of the uniformly distributed mass model depend on the total tension of the muscles of the hand-arm system (Figures 4 and 5).

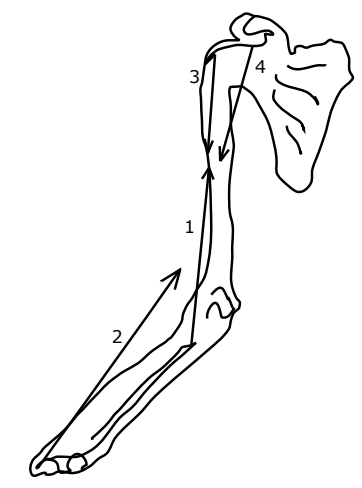

Figure 4. Flexor muscles

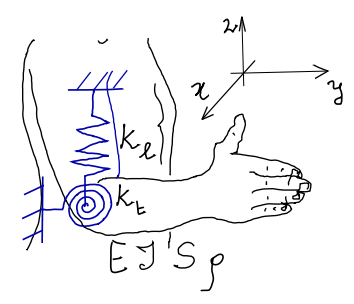

Figure 5. Hand arm system along the $\mathrm{x}, \mathrm{y}$ and $\mathrm{z}$ axes

The hand-arm system assumes an articulated configuration in working conditions. Solid hookean elastics, concentrated parameter models, Euler's beam model, and Timoshenko's beam model can represent mechanical models equivalent to the hand-arm system. The equivalence of the mechanical model concerns the physical quantities and the physical model of the hand-arm system configuration. The hand-arm system can be represented by the mechanical model in defined temperature, stress, and strain ranges.

The proposed model is an equivalent mechanical system that satisfactorily thickens the experimental values. The proposed model adopts a value of the Young module equivalent to the elasticity modulus. The hand-arm system with distributed parameters considers the phase speed and the group speed of the system response subjected to external excitation. Phase velocity is the velocity at which the mechanical wave phase propa- gates. The phase velocity represents the propagation velocity of a wave crest. Phase velocity differs from the velocity of signal propagation. The group velocity, or speed of wave amplitude variation, describes the propagation rate of the signal. The group velocity estimates the time variation of the envelope, modulating the wave.

A distributed parameter representation in the form of a beam model considers the continuous distribution of the mass, damping, and elasticity of the physical properties of the arm. The dynamic model of the human hand-arm is evaluated in the time domain. The effective beam length is the distance between the elbow and the center of the gripped handle. The wrist is included as part of the beam. If the hand is tightly gripping the handle, the wrist can become almost as stiff as the rest of the arm (Fig.5).

\subsection{Natural frequencies of human hand-arm system}

The differential equation of motion of beam is the following relation:

$$
\frac{\partial^{2}}{\partial x^{2}}\left[E I \frac{\partial^{2} w}{\partial x^{2}}\right]+\rho S \frac{\partial^{2} w}{\partial t^{2}}=0
$$

The solution of Eq.(1) can be obtained by the method of separation of variables

$$
\begin{aligned}
w(x, t)= & W(x) T(t) \\
= & \left(C_{1} \cos \beta x+C_{2} \sin \beta x+C_{3} \cosh \beta x+C_{4} \sinh \beta x\right) \\
& (A \cos \omega t+B \sin \omega t)
\end{aligned}
$$

where

- $C_{1}, C_{2}, C_{3}$ and $C_{4}$ are constants of integration;

- sinh and cosh are, respectively, the hyperbolic sine and cosine functions;

- the constant $\beta$ is the following relation:

$$
\beta^{4}=\frac{\rho S \omega^{2}}{E I}
$$

The natural frequencies of the beam are evaluated by the following expression:

$$
\omega=\beta^{2} \sqrt{\frac{E I}{\rho S}}
$$

Choosing the coordinate origin at the right end and substituting the following the boundary conditions at $x=0$ and $x=L$ :

- Free end

$$
\left(E I \frac{\partial^{2} w}{\partial x^{2}}\right)_{x=0}=0 \quad \text { (Bending Moment) }
$$

and

$$
\left[\frac{\partial}{\partial x}\left(E I \frac{\partial^{2} w}{\partial x^{2}}\right)\right]_{x=0}=0 \quad \text { (Shear Force) }
$$


Incidence of Predisposing Factors on the Human Hand-arm Response with Flexed and Extended Elbow Positions of Workers

- Elastically supported by linear spring. When the end of a beam undergoes a transverse displacement $\mathrm{w}$

$$
\left[\frac{\partial}{\partial x}\left(E I \frac{\partial^{2} w}{\partial x^{2}}\right)\right]_{x=0}=\left(k_{l} w\right)_{x=L} \quad(\text { Linear Spring })
$$

- Elastically supported by torsional spring, In this case the boundary condition is

$$
\left(E I \frac{\partial^{2} w}{\partial x^{2}}\right)_{x=L}=\left(k_{t} \frac{\partial w}{\partial x}\right)_{x=L} \quad \text { (Torsional Spring) }
$$

$\left[\begin{array}{cccc}0 & -\beta^{3} & 0 & \beta^{3} \\ -\beta^{2} & 0 & \beta^{2} & 0 \\ \beta^{3} \sin L \beta-k_{l} \cos L \beta & -k_{l} \sin L \beta-\beta^{3} \cos L \beta & \beta^{3} \sinh L \beta-k_{l} \cosh L \beta & \beta^{3} \cosh L \beta-k_{l} \sinh L \beta \\ \beta k_{t} \sin L \beta-\beta^{2} \cos L \beta & -\beta^{2} * \sin L * \beta-\beta * k_{t} * \cos L * \beta & +\beta^{2} * \cosh L \beta-\beta * k_{t} * \sinh L * \beta & \beta^{2} * \sinh L \beta-\beta k_{t} \cosh L * \beta\end{array}\right]\left\{\begin{array}{c}C_{1} \\ C_{2} \\ C_{3} \\ C_{4}\end{array}\right\}=0$

Expanding the frequency determinant, the characteristic equation becomes:

$$
\begin{aligned}
& (2 \beta+2 \beta \cos L \beta \cosh L \beta) k_{l} k_{t} \\
& +\left(2 \beta^{2} \cosh L \beta \sin L \beta-2 \beta^{2} \cos L \beta \sinh L \beta\right) k_{l} \\
& -\left(2 \beta^{4} \cos L \beta \sinh L \beta+2 \beta^{4} \cosh L \beta \sin L \beta\right) k_{t} \\
& +2 \beta^{5} \cos L \beta \cosh L \beta-2 \beta^{5}=0
\end{aligned}
$$

for free vibration frequencies.

Evaluation of constants yields

$$
\begin{aligned}
& C_{3}=C_{1} \quad C_{4}=C_{2} \\
& \frac{C_{1}}{C_{2}}=\frac{\beta^{3} \cos L \beta-\beta^{3} \cosh L \beta+k_{l}(\sin L \beta+\sinh L \beta)}{\beta^{3} \sin L \beta-k_{l}(\cos L \beta+\cosh L \beta)+\beta^{3} \sinh L \beta}
\end{aligned}
$$

The normalization of the mode shapes is with respect to the kinetic energy scalar product which yields

$$
\begin{aligned}
C_{j} & =\frac{1}{\sqrt{\int_{0}^{L}\left(\cos \beta_{j} x+\sin \beta_{j} x+\cosh \beta_{j} x+\sinh \beta_{j} x\right)^{2} d x}} \\
j & =1,2, \ldots
\end{aligned}
$$

\subsection{Forced vibrations of human hand-arm sys- tem}

The mode superposition principle offers the solution of forced vibrations

$$
w(x, t)=\sum_{n=1}^{\infty} W_{n}(x) q_{n}(t)
$$

where $W_{n}(x)$ is the normal mode functions and $q_{n}(t)$ is the generalized coordinate.

The steady-state response of the human hand-arm system is given by following relation

$$
q_{n}(t)=\frac{1}{\rho S b \omega_{n}} \int_{0}^{N} Q_{n}(\tau) \sin \omega_{n}(t-\tau) d \tau
$$

where $Q_{n}(\tau)$ is the generalized force corresponding to $q_{n}(t)$.

\section{Results}

Mathematical modeling and experimental investigation examine the dynamic behavior of the hand-arm system. The Table 1 illustrates the behavior of the roots deduced from the characteristic equation as a function of $k_{l}$ and $k_{t}$. The values of the natural frequencies of the hand-arm system depend on the values of the stiffness constants $k_{l}$ and $k_{t}$.

\subsection{Calibration of the mathematical model of human hand-arm system via Forward Re- gression}

The calibration of the mathematical model Eq.(8) of the human hand-arm system on experimental data used the forward regression. The principle is that the forward regression coefficient generates the best match between the results obtained by the proposed predictive model and the experimental data (Figures 6, 7, 8, 9 and 10), observed in Drill A, Drill B, Angle Grinder, Groover and Rotary Hammer.

$\mathrm{R}$-square, adjusted R-square, root mean squared error evaluated the goodness-of-fit or the discrepancy between measured accelerations and predicted ones (Table 2).

The R-squared coefficient of determination showed the variation of the mathematical model response to the independent variables in the linear regression model. The high $\mathrm{R}-$ squared values of $0.88-0.97$ showed that the mathematical model was very well adapted to experimental investigations.

Adjusted $\mathrm{R}^{2}$ represented a refinement of the goodness of fit. Adjusted $\mathrm{R}^{2}$ included a penalty for the number of terms in the mathematical model. Adjusted $\mathrm{R}^{2}$ reached high values in the $0.89-0.98$ range, indicating the agreement between the accelerations obtained by the mathematical model and experimental ones.

The root mean squared error (RMSE) quantified the error between the accelerations predicted by the mathematical model and the acceleration observed. The root mean squared error reached smaller values of less than 0.09 . 
Table 1. Natural frequencies of human hand-arm system in function of $k_{l}$ and $k_{t}$

\begin{tabular}{ccccc}
\hline Natural Frequencies & \multicolumn{4}{c}{$k_{t}[\mathrm{~N} / \mathrm{m}]$} \\
\cline { 2 - 5 } & 0.001 & \multicolumn{4}{c}{1} & 10 & 100 \\
$\mathrm{~Hz}$ & \multicolumn{4}{c}{$k_{l}[\mathrm{~N} / \mathrm{m}]$} \\
\hline 2.4 & 1.90 & 6.11 & 7.48 & 7.66 \\
3.8 & 4.74 & 12.37 & 18.57 & 19.78 \\
132.9 & 2859 & 2911.7 & 3187.2 & 2500.8 \\
\hline
\end{tabular}

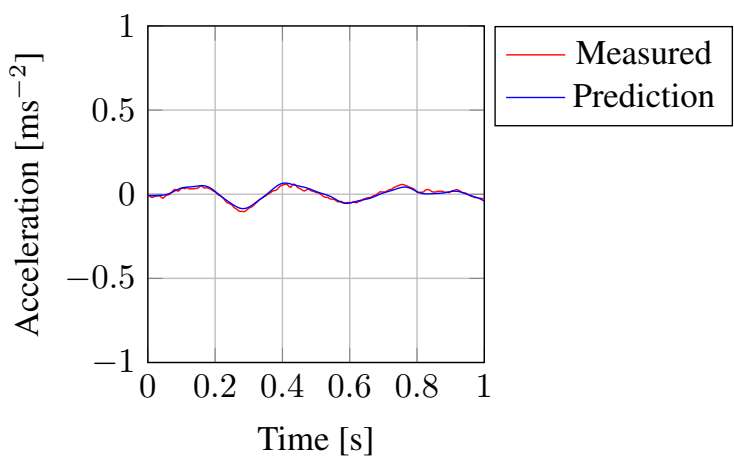

Figure 6. Predicted vs. Observed values in Drill A

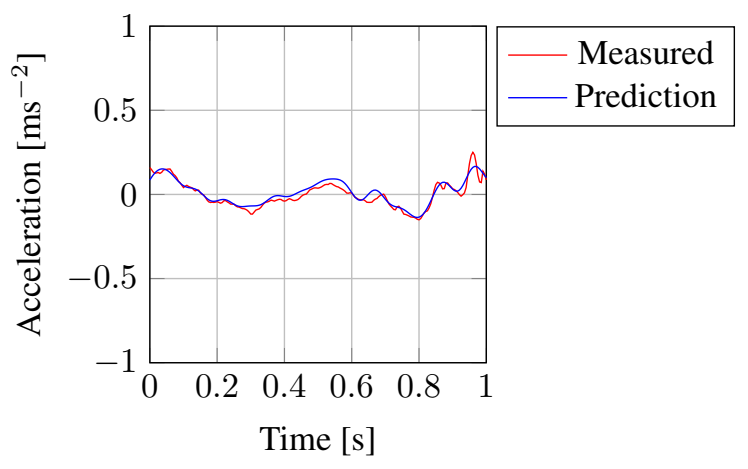

Figure 7. Predicted vs. Observed values in Drill B

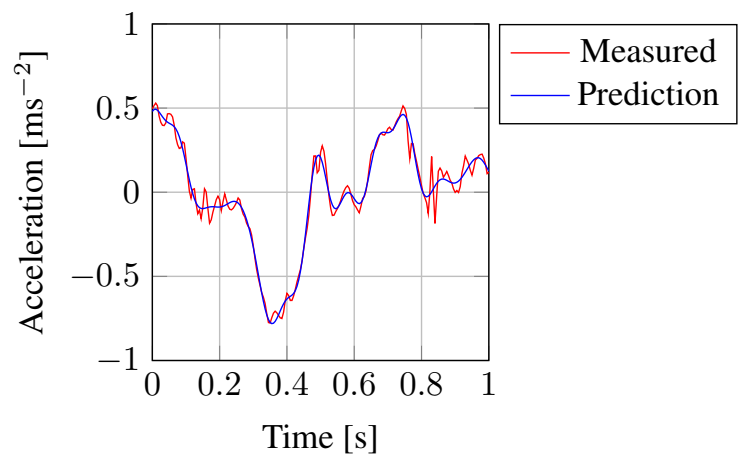

Figure 8. Predicted vs. Observed values in Angle Grinder

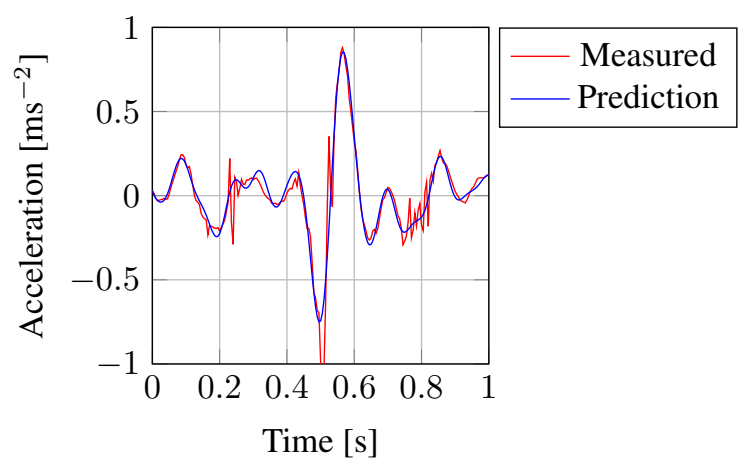

Figure 9. Predicted vs. Observed values in Groover

\subsection{The characteristics of vibration accelera- tion signals}

The features of vibration acceleration signals are Peak, Peak-to-Peak, Max, RMS values along $\mathrm{x}, \mathrm{y}$, and $\mathrm{z}$-axis (Figures 11, 12, 13 and 14). The mechanical characteristics of machine tools are the number of revolutions, weight, power, and diameter of the handle (Table 3). The measurements were carried out by Svantek SV 106A six-channel Human Vibration Meter and Analyser according to ISO 8041-1:2017, ISO 2631-1, 2-5, ISO 5349, and directive 2002/44/EC of European Parliament.

The experimental investigation offers the correlation matrices concerning the features of the vibratory signals and respect to mechanical characteristics of machine tools (Figures 15, 16, 17 and 18).

The biodynamic response of the human hand-arm system is frequency-dependent. The resonant frequencies of the human arm system are frequencies at which the oscillation of the tissues is amplified. The resonant frequencies of the human arm system are between 1 and $130 \mathrm{~Hz}$. For against, the resonant frequencies of individual fingers are in the field of $150-300 \mathrm{~Hz}$. Vibrations at frequencies greater than $100 \mathrm{~Hz}$ are transmitted to the tissues of the fingers and hands. Also, vibrations at frequencies greater than $100 \mathrm{~Hz}$ are not transmitted to the rest of the hand-arm system. The experimental investigation and the prediction of the biodynamic response of the human and arm system to vibration sources are critical for understanding how mechanical vibrations may be compensated by muscle actions. The frequency range of the prevailing forced vibrations is $0-85$ $\mathrm{Hz}$ (Fig. 19 and 20). 


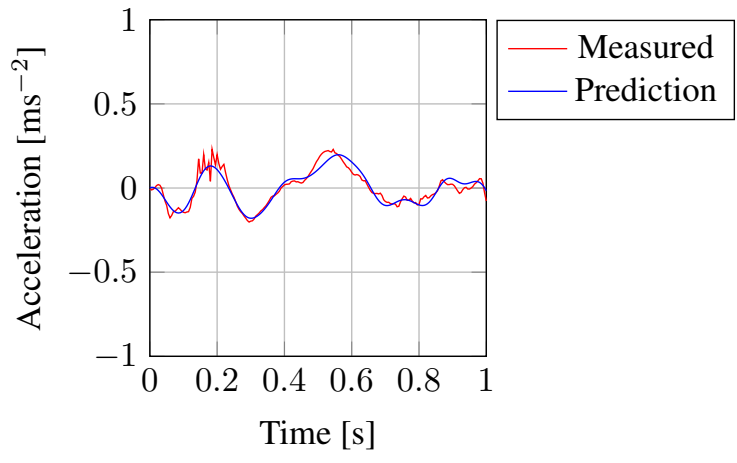

Figure 10. Predicted vs. Observed values in Rotary Hammer

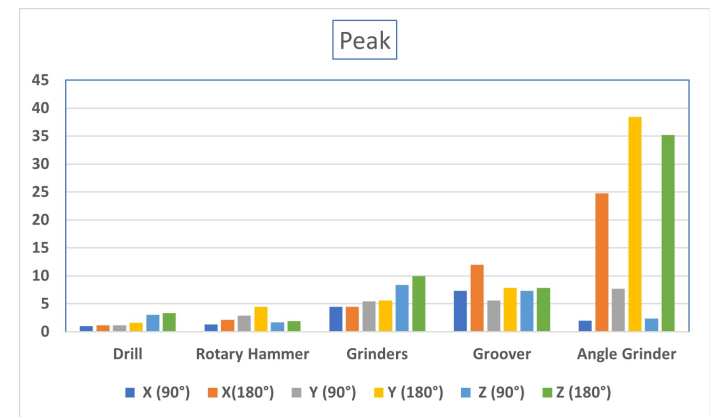

Figure 11. Peak values

\subsection{Comparison between dominant hand and non-dominand hand}

Professional machining tools are electric, battery, pneumatic, and combustion. All work instruments have a percussion or rotating mechanism and can transmit high mechanical vibration values to the hand-arm system, increasing the incidence of disturbances. Working tools can generate a wide variety of even very serious disorders. Generally, the right hand is preferred to handle some tools and the left hand has a different function. In many cases, the dominant hand grabs the handle of the instrument and controls the trigger of the professional instrument. The dominant hand represents the human hand that workers prefer to use when performing the work gesture. The dominant hand presents faster and more precise movements. The dominant hand shows a better control of the working gesture. The non-dominant hand shows less control

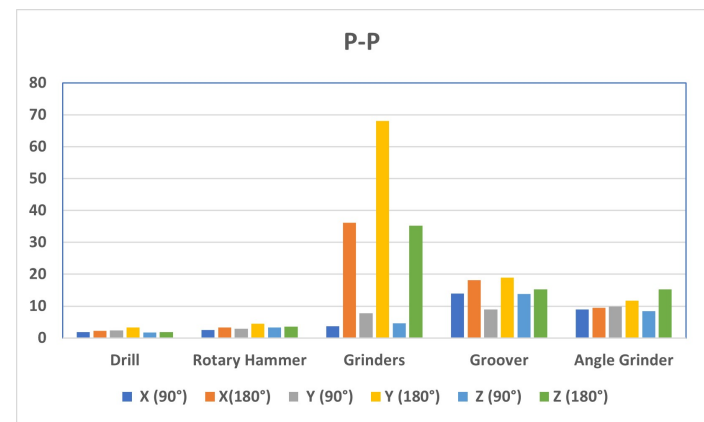

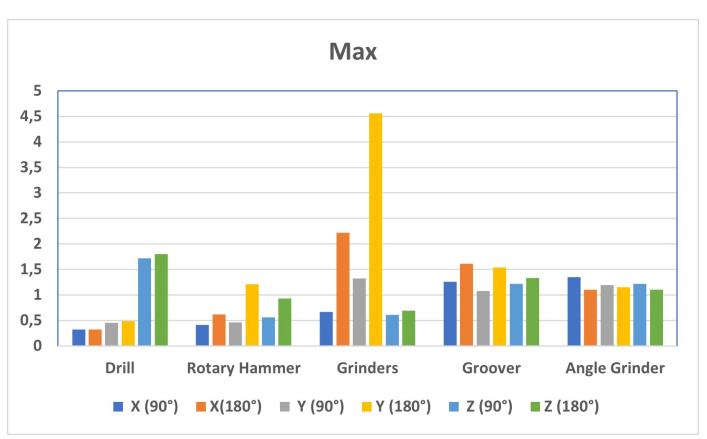

Figure 13. Max values

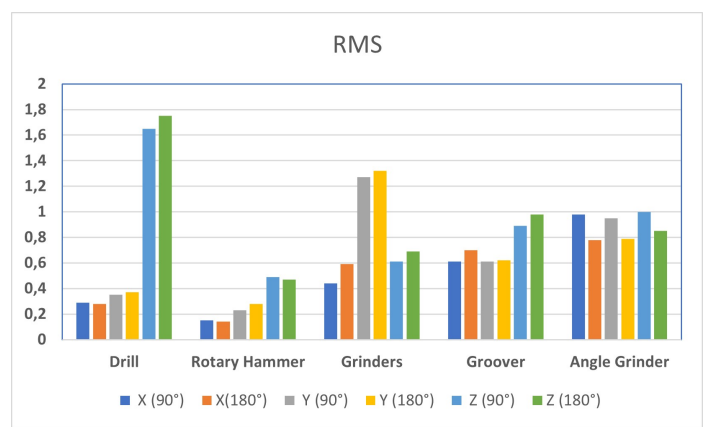

Figure 14. Root mean square values



Figure 15. Correlation matrix between mechanical characteristics of machine tools and peak of time response 


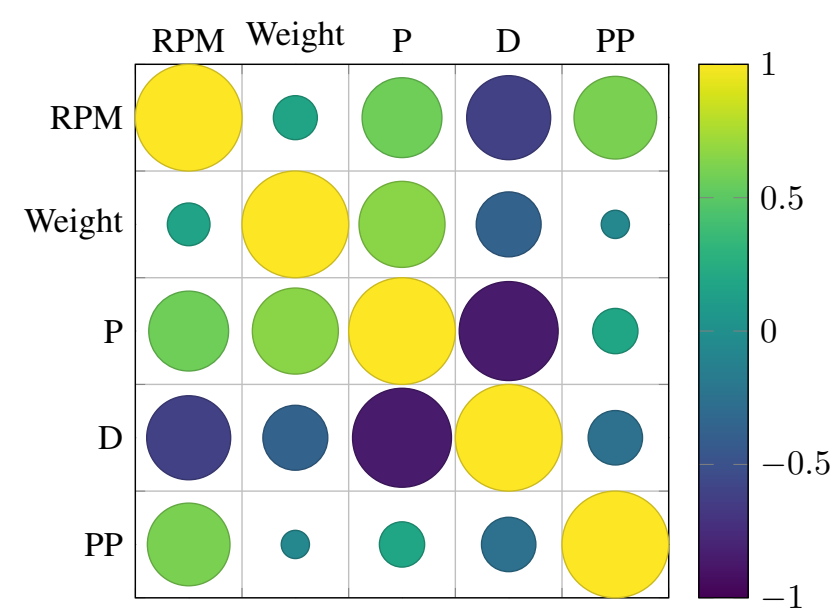

Figure 16. Correlation matrix between mechanical characteristics of machine tools and peak-peak of time response

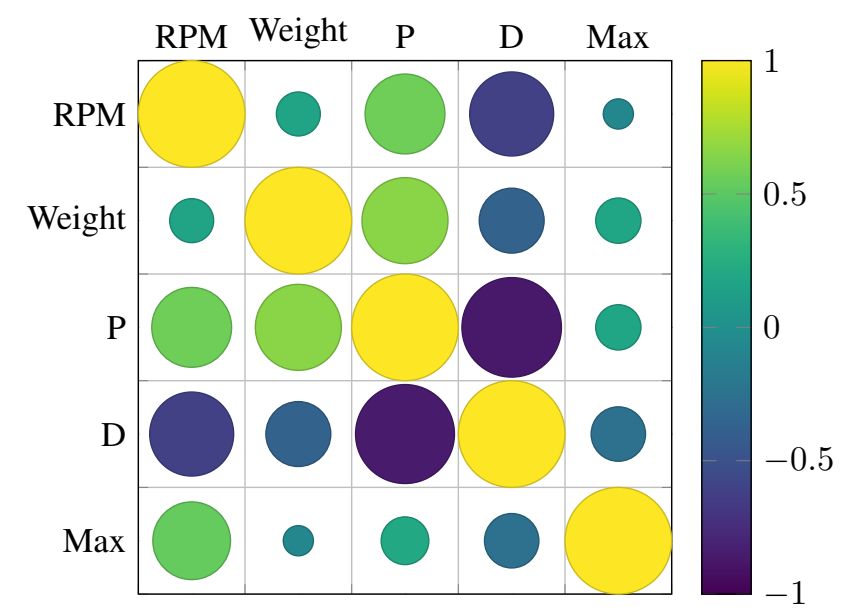

Figure 17. Correlation matrix between mechanical characteristics of machine tools and max of time response

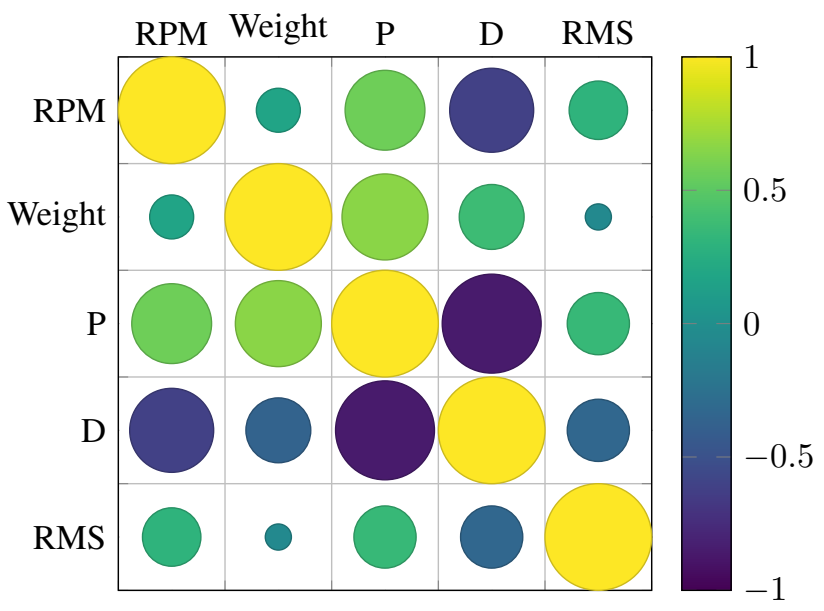

Figure 18. Correlation matrix between mechanical characteristics of machine tools and RMS of time response

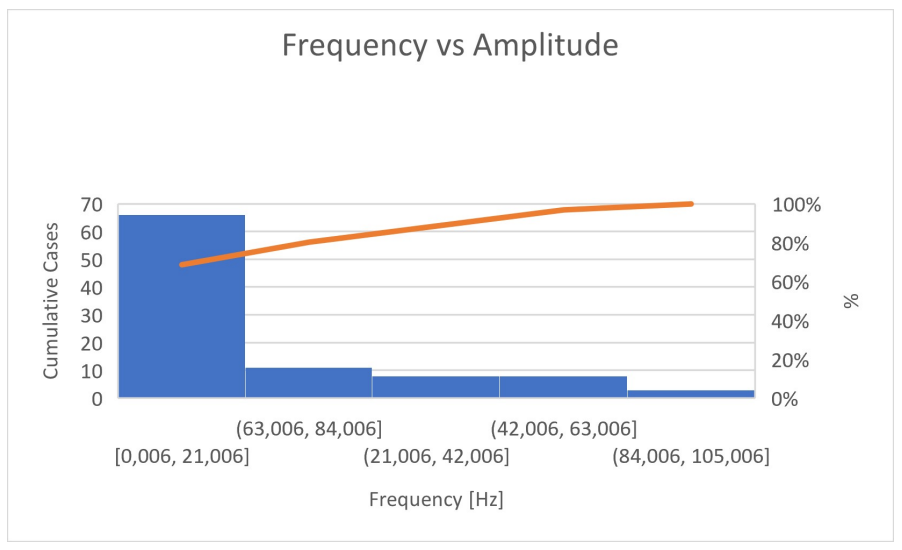

Figure 19. Amplitude vs Frequency

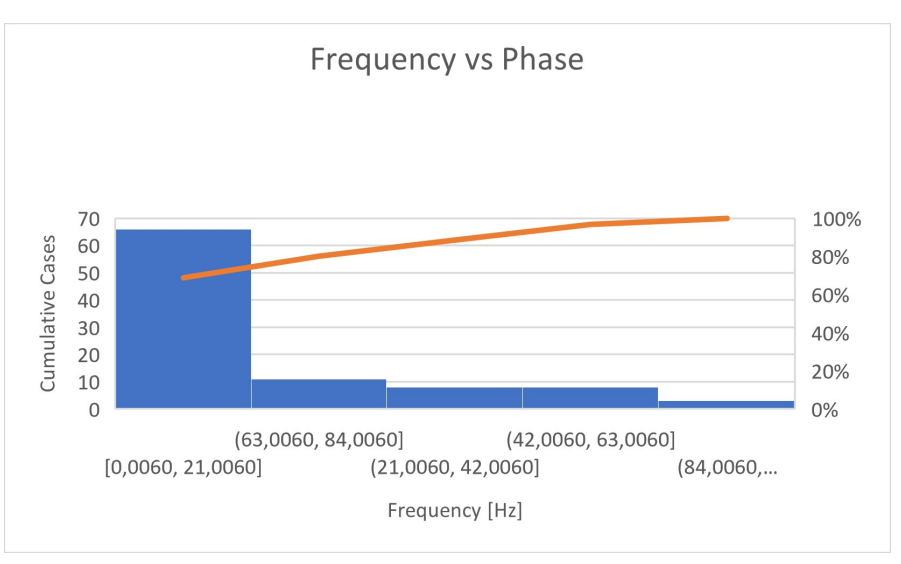

Figure 20. Phase vs Frequency 
of the work gesture. The dominant hand muscles are stronger and have greater dexterity, while they are less developed in the less dominant hand. A dominant hand is about $10 \%$ stronger when grabbing objects than a non-dominant hand.

If the non-dominant hand does not always grasp the instrument, this dominant may perform other functions. The nondominant hand can guide another tool needed to control or facilitate machining; it can control the tool itself; it can help to carry out machining according to the skill of the worker. Each worker may be exposed to mechanical vibration according to $\mathrm{x}-, \mathrm{y}-$, and $\mathrm{z}-\mathrm{axes}$. The location of the measuring points should be chosen concerning the manual skill of the worker. The location of the acceleration measurement points depends on the machining. The rotating tools, used for metal processing, operate between $6,000-11,000$ rpm [10]. Percussion instruments can generate high-intensity shocks. Mechanical shocks contain a high energy content in a wide frequency band. Therefore, shocks generate dangerous mechanical vibrations first on the tool and then on the hand-arm system in a wide range of frequencies. The use of mechanical filters mitigates the high amplitudes of unwanted mechanical vibration frequency components. Measurements are taken on the handles and other parts of the instrument with screwed, glued, or locked accelerometers in the appropriate position.

\subsection{Acceleration spectra}

The acceleration spectra in the octave band are obtained according to the three axes on the handle under numerous working conditions using experimental investigations. The total frequency weighted accelerations, acquired on the instrument handle, are expressed in $\mathrm{m} / \mathrm{s}^{2}$ r.m.s. according to the $\mathrm{x}-, \mathrm{y}-$, and $\mathrm{z}$-axes of the human hand-arm system [10].

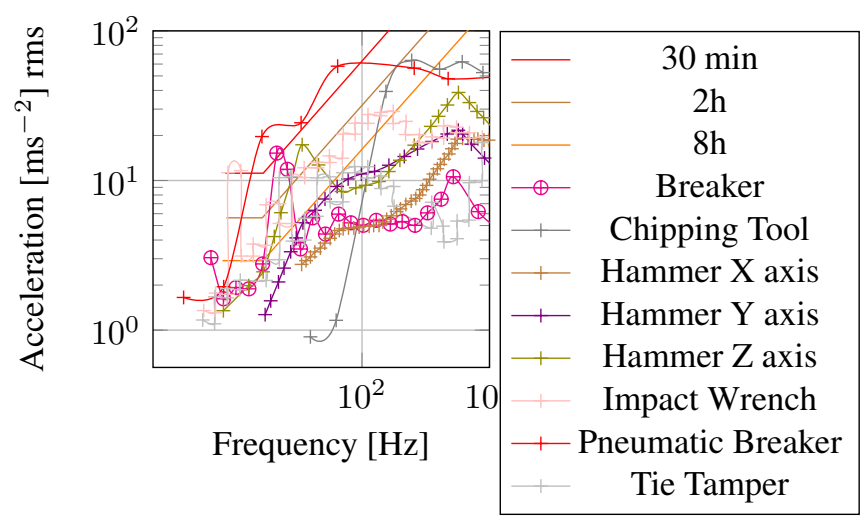

Figure 21. Octave band acceleration spectrum measured on handle of Breaker, Chipping Tool, Hammer $\mathrm{X}$ axis, Hammer $\mathrm{Y}$ axis, Hammer $\mathrm{Z}$ axis, Impact Wrench, Pneumatic Road Breaker, Tie Tamper compared to 8h, 2h and $30 \mathrm{~min}$ exposures

Following regulatory standards, only the dominant axis is considered. Daily exposure to mechanical vibrations is compared with the recommended action level [10]. The test procedure reduces variability in measurements. However, different operators and different laboratories can obtain very different measurements. Laboratory measurements may be different

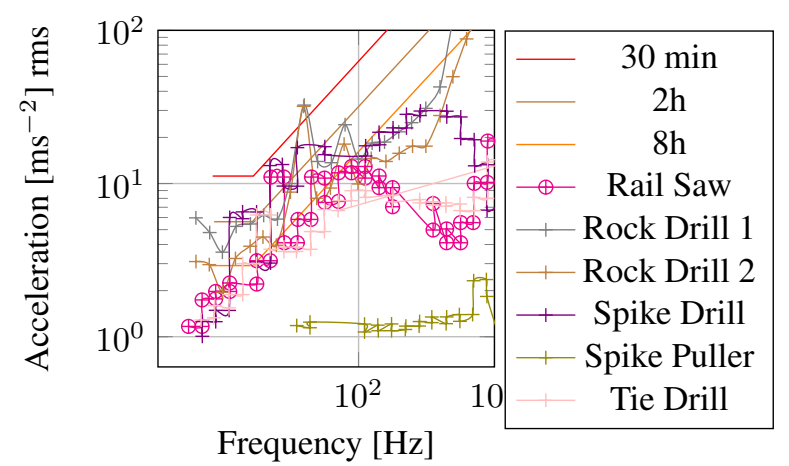

Figure 22. Octave band acceleration spectrum measured on handle of Rail Saw,Rock Drill 1, Rock Drill 2, Spike Drill, Spike Puller compared to 8h, 2h and $30 \mathrm{~min}$ exposures

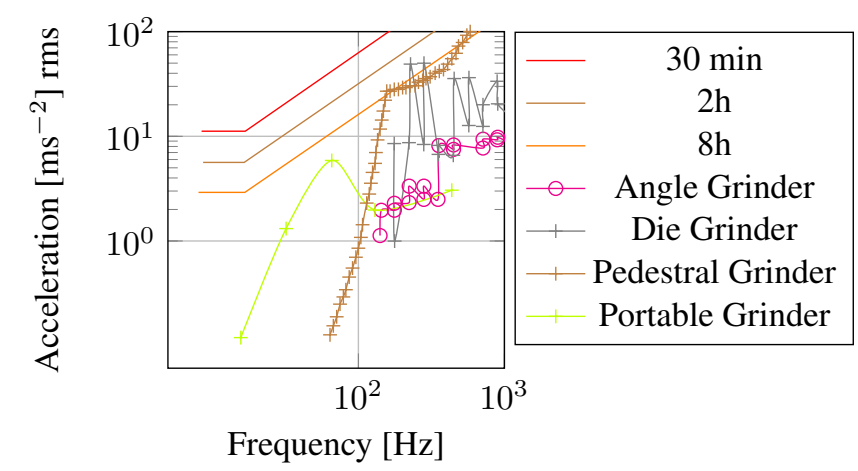

Figure 23. Octave band acceleration spectrum measured on handle of Angle Grinder, Die Grinder, Pedestral Grinder,Portable Grinder compared to 8h, $2 \mathrm{~h}$ and $30 \mathrm{~min}$ exposures



Figure 24. Octave band acceleration spectrum measured on handle of Chainsaw 1, Chainsaw 2, Chainsaw 1972, Chainsaw 1982 compared to 8h, 2h and 30 min exposures 
from measurements on workers using instruments for work activities. Figures 21, 22, 23 and 24 illustrate the trend of acceleration in the frequency domain. The harmonic components of the forced response are compared with the natural pulsations of the hand-arm system.

The handle of a tie tamper is connected to the engine that generates vibrations. The tie tamper in question activates an integral batter. The tie tamper could be used just under 1 hour a day. Industrial innovation has produced tools with springloaded anti-vibration grip. The anti-vibration handle isolates the vibrations of the tool to reduce operator fatigue and increase operator productivity (Fig.21).

The hydraulic spike puller introduced an automatic cycle to reduce recoil and improve usage. The handle of the hydraulic spike puller ensures high ergonomics. The lateral transport handle of the hydraulic spike puller allows easy use. The spectrum of accelerations shows that the measurement has not exceeded the limit values. The tested spike puller can be used up to eight hours/day (Fig.22).

Inadequate maintenance can cause some mechanical vibration problems. An unbalanced rotating component can generate mechanical vibration. The spike drill tool, grip with the right hand, exceeds the limit values according to the $\mathrm{x}$-axis.

Ergonomic handles and an anti-vibration system allow the operator to make quick and easy cuts with less effort. But the cutting is done according to $\mathrm{x}, \mathrm{y}$, and $\mathrm{z}$ directions. In the case of the rail saw tool, the standard has been exceeded along the $\mathrm{X}$-axis.

A rotating mass of the impact wrench stores energy. Such rotating mass is released instantly to provoke impacts on the rotating shaft. The impact produces a high force on the bolt or nut to tighten or unscrew. A spring pushes the shaft to provide a high force. Energy dissipation occurs in a short time. The tested impact wrench should be used for less than 30 minutes/day (Fig.21).

The use of brushless motors overcomes the drawback that a tie drill should be used less than one hour/day. The Brushless motor can produce a high torque value. A higher torque available requires less power. The efficiency of the engines increases, the overall life of the products is prolonged, maintenance becomes simpler and the comfort of the worker increases.

The workers need to know the risks and prevention techniques to reduce the risk of vibration. Prevention means that a machine does not present limitations or dangers to the workers. Some machining operations must be carried out at high tool rotation speeds. The die grinder has a rotation speed of 18,000 $\mathrm{rpm}$. The mechanical vibrations of the die grinder exceed the recommended limit of 4-8 hr/day for the y measurement direction (Fig.23).

The frequency-domain acceleration diagram reveals that electric rock drills and chainsaws contain frequency components that differ greatly in amplitude. The peak of the acceleration spectrum of the chainsaw occurs at about $160 \mathrm{~Hz}$. The peak acceleration spectrum of rock drills occurs at about 800 $\mathrm{Hz}$ (Fig.24).

Operators may perform incorrect manoeuvres. Workers may have limited working space. There are many scenarios of work conditions. Untrained workers lifted the breaker without turning it off. Untrained workers or workers with little working space increase the risk of mechanical vibrations. Untrained workers operate uninterruptedly for about a minute without moving the cutting tool. The pavement breaker spectrum presents two peaks, one at about $20 \mathrm{~Hz}$ and a second at about $1250 \mathrm{~Hz}$. The first peak is close to the natural frequencies of the hand arm system (Fig.21).

Jackhammers, breakers, scalers, rotary hammers, hammer drills, jumping jacks, and other compactors, manifest problems of mechanical vibrations within just a half-hour of work. The manufacturers adopt a counterweight driven in the opposite direction to the striker to reduce the action of the vibratory force. The action of counterweight balances the instrument and reduces the impact force and overall vibration.

Hammers and percussion drills, jack drills, rock drills used in a horizontal direction, drills, and hammers for breaking, chipping, and removing material in mines and quarries, and breakers cause vibration injuries. Pneumatic breakers and hydraulic breakers operate on concrete and asphalt. The action on concrete and asphalt generates reactions with high amplitudes. Road work is dominated by low-frequency vibrations. The worker, who uses the breaker on road surfaces, can change the hand of the handle and guide the breaker through the handle, keeping the tip of the instrument resting on the ground. Hammers and percussion drills generate a high incidence of injuries to bones and joints, causing vascular disorders in the fingers of both hands. The vibration of road breakers can cause white fingers induced by vibration (VWF). The octave band spectra of a hammer show the maximum vibration around the band of $31.5 \mathrm{~Hz}$. The spectrum shown in Fig. 21 has the maximum weighted acceleration amplitude of $29.5 \mathrm{~m} / \mathrm{s}^{2}$ root mean square (r.m.s.). The action level can be exceeded after few minutes of exposure to mechanical vibrations. The measurements indicate that the worker suffers short and irregular daily exposure to mechanical vibrations of road breakers. The worker may suffer important symptoms. The total daily exposure time may be greater than 30 minutes.

The use of grinders causes severe exposure to mechanical vibrations in grinding operations. All grinding operations of small or large objects cause exposure to mechanical vibrations. Exposure to mechanical vibration depends on the material, shape, and mass of the object to be rectified, the type, condition of the grinding wheel, and the operator's operating technique. If the vibration intensity depends on the type of object to be worked on, the wear conditions of the tools assume a significant rule. If the worker works with a worn-out grinding wheel, unwanted mechanical vibrations arise. A single grinding process can last a few seconds. But the daily exposure of the worker results from many operations of short intervals of time. The measurement protocol provides for the attachment of the accelerometers to the work instruments through a frame held in the hand. The worker may develop his operational skills according to the measurement protocol. The action level can be exceeded after a few minutes of exposure even in a single direction. Instruments have been designed to minimize the effects of grinding.

The motor and the interaction of the chainsaw chain with 
Incidence of Predisposing Factors on the Human Hand-arm Response with Flexed and Extended Elbow Positions of Workers 516 Subject to Different Sources of Vibrations

the wood to be cut generate mechanical vibrations. Chainsaws can be equipped with two-stroke single-cylinder engines, less balanced than multi-stroke four-stroke engines. The counterweights on the crankshaft of single-cylinder engines balance the forces of reciprocating piston movement. Chainsaw handles can transmit mechanical vibrations. Gasoline chainsaws have a vibro insulation system to isolate the effect of the drive units. The handles of the chainsaws have rubber springs to reduce mechanical vibrations. Electric chainsaws do not have a vibration reduction system. Chainsaw handles can be connected directly to the drive unit, mechanical vibration source.

\section{Discussion}

The primary extensor muscles of the upper extremities (Fig.4) are the following muscles:

- brachialis (1) acts exclusively as the flexor of the elbow;

- brachio-radialis (2) acts essentially as a flexor;

- biceps brachii $(3,4)$ represents a biarticular muscle with the function of elbow flexor muscle.

If the hand-arm system is subject to mechanical vibrations along the $\mathrm{x}, \mathrm{y}$, and $\mathrm{z}$-axes (Fig.5), the aforementioned flexor muscles work at their best advantage when the elbow is flexed at $90^{\circ}$.

If the arm is extended (Fig.1) the direction of the forces exerted by the muscles is almost parallel (white arrow) to the axis of the lever arm. The centripetal component $\mathrm{C}$ acts in the direction of the center of the joint. Component $\mathrm{C}$ is powerful but not very effective in flexion actions. The weak transverse component $\mathrm{T}$ is the only effective force in elbow flexion. The flexion angle of maximum efficiency is between $80^{\circ}$ and $90^{\circ}$ (Fig.2) for the biceps and the brachioradialis between $100^{\circ}$ and $110^{\circ}$ (Fig.3). The action of the flexor muscles follows the physical laws of the levers of the third type and promotes a good range and speed of movement with little power.

The models exhibit natural frequencies the mode shapes in the $0-130 \mathrm{~Hz}$ range.

If $k_{t}=0.001$, the characteristic equation has the first natural frequency $f_{1}=2.4 \mathrm{~Hz}$ with $k_{l}=1.90 \mathrm{~N} / \mathrm{m}$. The second natural frequency is $f_{1}=3.8 \mathrm{~Hz}$ with $k_{l}=4.74 \mathrm{~N} / \mathrm{m}$. The third natural frequency, deduced from the characteristic equation, is $f_{3}=132.9 \mathrm{~Hz}$ with $k_{l}=2859.0 \mathrm{~N} / \mathrm{m}$. The stiffness values $k_{l}$ are deduced assuming the following numerical values $E I=3.8 \mathrm{~N} \mathrm{~m}^{2}, \rho=3.31 \mathrm{~kg} / \mathrm{m}$ and $L=0.38 \mathrm{~m}$. The linear stiffness, offered by the action of the extensor muscles, increases with an increment of the order of natural frequency of the human hand-arm system.

The effects of mechanical vibrations of 5 machine tools on the hand-arm system are examined along the $\mathrm{x}, \mathrm{y}$, and $\mathrm{z}$ axes (Fig.5). Vibratory signals are evaluated in the time domain. The measurements have been repeated many times to increase confidence in experimental investigation. The features of the
The features of vibration acceleration signals are Peak, Peakto-Peak, Max, RMS values. The mechanical characteristics of machine tools are the number of revolutions, weight, power, and diameter of the handle. The variation ranges are the following fields: number of revolutions RPM=550-11000 $\mathrm{min}^{-1}$; Weight $=1.84-5.6 \mathrm{~kg}$; Power $\mathrm{P}=1.0-2.5 \mathrm{~W}$; diameter $\mathrm{D}=0.025-$ $0.045 \mathrm{~m}$ (Table 3).

The research proposes the normalized correlation matrices between the magnitudes of mechanical vibrations and mechanical characteristics of machine tools. The correlation is 0.6 between the RPM of the machine tools and the peak. The correlation is 0.6 between the number of revolutions of the machine tools and the peak-to-peak of the vibratory signals in the time domain (Figures 15, 16, 17 and 18).

If the worker uses the angle grinder tool with the extended arm configuration, the peak values present elevated levels along the $\mathrm{x}, \mathrm{y}$, and $\mathrm{z}$-axes. The highest peak value is recorded along the $y$ axis (Fig.11).

The grinders, used in the extended arm configuration, offer high peak-to-peak values along the $\mathrm{x}, \mathrm{y}$, and $\mathrm{z}$-axes. The highest peak-peak value is acquired along the y-axis (Fig.12).

The drill tool in the extended elbow configuration offers high MAX values along the $\mathrm{x}-$ and $\mathrm{y}$-axes. The highest value of MAX has been acquired according to the $\mathrm{z}$-axis (Fig.13).

The drill tool, used in bent and extended elbow configuration, offers high RMS values along the $\mathrm{z}$-axis. In the case of the drill tool in the extended elbow configuration, the highest RMS value is acquired along the $\mathrm{z}$-axis (Fig.14).

The efficiency of the triceps depends on the state of flexion or extension of the elbow. In full extension (Fig.1) the muscular force can generate two components: the radial or centrifugal component $\mathrm{C}$ and normal (tangential) component $\mathrm{T}$. The centrifugal component $\mathrm{C}$ tends to dislocate ulna posteriorly. Component $\mathrm{T}$ is a more powerful normal (tangential) component. Component $\mathrm{T}$ is the only active force in extension.

If the elbow is moderately flexed to $20^{\circ}$ to $30^{\circ}$ the radial component becomes zero and the effective tangential component $\mathrm{T}$ generates the muscular pull. In this position, the triceps are very efficient (Fig.2).

If the elbow is flexed further, the effective tangential component $\mathrm{T}$ decreases, and the centripetal component $\mathrm{C}$ increases (Fig.2).

In full flexion, the triceps tend to control their loss of efficiency. Moreover, the fibers of the triceps are maximally stretched. The force of contraction of the triceps is maximal to decrease its loss of efficiency (Fig.3).

The measurements on the work conditions differ from laboratory measurements. The aspects examined concern grip, posture, and reduction of strength grip. In the laboratory, the worker assumes a static posture. For against, the worker, in the job conditions, assumes a dynamic posture, rarely static posture. During movement, the muscles shorten and lengthen, the tendons slide against their sheaths, the nerves articulate through their sheaths, the joint surfaces slip and roll over each other and the fluid flow increases in most tissues. The running speed of the tendons, in the respective sheaths under load, generates a work of friction. The work generated by the friction force depends on the frequency, the human hand force, and the 
Table 2. Goodness-of-Fit Statistics

\begin{tabular}{cccc}
\hline Tool & R-squared & Adjusted R & RMSE \\
\hline Drill A & 0.9344 & 0.9258 & 0.0103 \\
Drill B & 0.8826 & 0.8674 & 0.0290 \\
Angle Grinder & 0.9774 & 0.9745 & 0.0516 \\
Groover & 0.9105 & 0.8989 & 0.0847 \\
Rotary Hammer & 0.9060 & 0.8938 & 0.0341 \\
\hline
\end{tabular}

Table 3. Mechanical characteristics of machine tools

\begin{tabular}{llcccc}
\hline Tool & Tool Movement & $\begin{array}{c}\text { RPM } \\
{\left[\mathrm{min}^{-1}\right]}\end{array}$ & $\begin{array}{c}\text { Weight } \\
{[\mathrm{kg}]}\end{array}$ & $\begin{array}{c}\mathrm{P} \\
{[\mathrm{kW}]}\end{array}$ & $\begin{array}{c}\mathrm{D} \\
{[\mathrm{m}]}\end{array}$ \\
\hline Drill & rotation & 550 & 1.84 & 1.0 & 0.045 \\
\hline Rotary Hammer & rotation & 750 & 4.2 & 0.85 & 0.045 \\
\hline Grinders & straight-axial, vertical, rotation & 11000 & 2.4 & 1.4 & 0.035 \\
\hline Groover & rotation & 7500 & 5.6 & 1.95 & 0.040 \\
\hline Angle Grinder & rotation & 6500 & 5.1 & 2.5 & 0.025 \\
\hline
\end{tabular}

deviation of the wrist from the neutral position. The movement of the human hand generates speed and acceleration on the various parts of the body. Increased angular acceleration on the wrist increases the risk of accumulation of trauma disorders. The increase of the risk provokes an increment of the loads on the tendons and other structures involved in the accelerations of the hand. If the movement characterizes the posture of the worker, the grip presents two phases. The worker performs two different types of grip (grasping): power take and precision grip. In the first grip, the worker grabs his work tool holding it totally inside the compass of the hand. In the second grip, the worker grabs the object with a precision grip. The worker carries out the machining process using power take and precision grip. The worker develops an operational ability, where the action of external forces interacts with the effects of posture [10].

Movements generate combined effects of external forces and postures. Combined effects affect comfort and performance. The misalignment between the center of the hand and the center of the wrist represents a posture of the arm-hand system. In this posture, an external force on the sides of the fingers compresses the nerve and blood vessels. The force of gravity acts on the center of the mass of the hand. The force of gravity, applied to the center of the hand, generates a radial moment of the wrist. The deviation of the wrist from the neutral position increases the pressure on the carpal canal. The deviation of the wrist creates loads on the tendons. The movement of the hand and wrist affects the movement of the elbow. The support on the elbow can be an aid for the worker. But possible elbow support compresses the nerve. During the grip, elbow, wrist, and finger postures interact together and generate other handarm system configurations: pronation/supination of the forearm, wrist ulnar/radial deviation, and wrist extension/flexion. The conclusions are similar. Pronation/supination of the forearm increases the pressure on the carpal tunnel. Similarly, the wrist ulnar/radial deviation and the wrist extension/flexion generate pressure on the carpal tunnel. The musculoskeletal system responds synergistically to the different configurations of the hand-arm system. The synergy is due to the action of multiple muscles. The muscles support and extend the joints according to the requirements of working conditions. Despite the synergistic action of the musculoskeletal system, the pressures of the carpal canal remain influenced by the rotation of the forearm and by the metacarpal-phalanx flexion. Portable vibrating instruments cause other vibration syndromes on the workers' hand-arm. White fingers, widely distributed finger neuropathy, pain in the arm and hand, risk of osteoarthritis, percussion in the wrist and elbow represent further damage caused by mechanical vibrations. The already mentioned syndromes can also be caused by ergonomic factors other than vibrations. Mechanical vibrations are the most important cause of the development of carpal tunnel syndrome, but not the only cause.

Measurements of accelerations allow us to investigate the effects and consequences of posture interactions. Deleterious activity includes physical discomfort beyond the carpal tunnel pressure. Deleterious activity can amplify some physical discomfort and the consequences on carpal tunnel pressure. The pronation/supination of the forearm generates reduced comfort, reduced grip of force, then further increase of the pressure on the carpal tunnel. Similarly, the wrist ulnar/radial deviation and the wrist extension/flexion generate reduced comfort, reduced grip force, then increased pressure on the carpal tunnel.

As previously mentioned, performance aspects, influenced by arm posture, include tension, strength, endurance, activity time, and reduction of grip force. The grip force depends on the posture of the worker. The pressure of the carpal canal increases with the postural deviations according to the rectilinear configuration of the wrist. The applied load increases the pressure of the carpal tunnel. The posture, identified by the joint angles, affects comfort. The postures of the elbow, forearm, 
Incidence of Predisposing Factors on the Human Hand-arm Response with Flexed and Extended Elbow Positions of Workers 518 Subject to Different Sources of Vibrations

and wrist influence effective and healthy performance. Performance, comfort, and risk of musculoskeletal injuries are the criteria for evaluation. Three comfort regions can be identified: a neutral region that represents the minimum discomfort to the joint and adjacent structures; a region of effort defined as an effort associated with medium discomfort; a maximum value defined as the recommended limit. Prolonged postural loads result in static muscle activity, which can cause muscle pain. The evaluation of comfort is associated with the efficiency of performance. The region of maximum force for the power grip is concerning the following range: wrist extension $35^{\circ} \pm 2^{\circ}$; ulnar abduction of the wrist $7^{\circ} \pm 2^{\circ}$; elbow flexion from $65^{\circ}$ to $100^{\circ}$. Correct postures limit stresses by a local contact in the following regions: center of the hand, fingers, thumb, elbow, and carpal canal region. The correct postures avoid pressure on the nerves and blood vessels inside the elbow.

The comfort assessment is evaluated by testing within a time interval of 60 seconds. Experimental data may provide an assessment of the comfort of different joints. But the time interval required to perform the work may be longer than the time interval chosen for the tests. If the posture is maintained for a longer period, the posture, already classified good, can generate strong discomfort and decrease the strength of the worker.

This research considers the interaction of elbow, wrist, and finger postures. The distal musculoskeletal system at the elbow works synergistically during the grip. The synergy is due to the action of multiple muscles that adapt to the needs of the task. The posture of the elbow, the posture of the wrist, and the posture of the fingers are related to each other. The forearm rotation and the metacarpal-phalanx flexion influence the pressures of the carpal canal. This research assesses the cause/effect relationships, the link between mechanical vibration, actual exposure, and the worker's symptoms. The exposure assessment shall consider the nature of the mechanical vibrations, the duration, and intermittence of the work. The experimental investigation data offers the ranges of the wrist, forearm, and elbow movement intervals. This research collects epidemiological data on the relationships between mechanical vibrations and excessive risks of white fingers and other neuropathy. External forces, transmitted through the skeletal connection concerning postural effects, provoke forces in the tissues. The comfort and risk of injury depend on the variation in the time of the forces. Posture, therefore, is a peculiar aspect of healthy and effective activity.

Prescribed Vibration White Finger (VWF) and Carpal Tunnel Syndrome (CTS) in Great Britain are monitored since 1995. Le prescrizioni registrano un'evoluzione continua dal 1985. Since 1st Aprile 1985, the prescription concerns vibration effects as induced white finger episodic blanching, occurring throughout the year, affecting the middle or proximal phalanges (or in the case of the thumb the proximal phalanx) of any three fingers. Occupations are the following ones:

1. the use of handheld chainsaws in forestry; or

2. the use of handheld rotary tools in grinding, or in sanding or polishing of metal, or the holding of material being ground, or metal being sanded or polished, by rotary tools; or
3. the use of handheld percussive metal-working tools, or the holding of metal being worked upon by percussive tools, in riveting, caulking, chipping, hammering, fettling, or swaging; or

4. the use of handheld powered percussive hammers in mining, quarrying, demolition, or on roads or footpaths, including road construction; or

5. the holding of material being worked upon by pounding machines in shoe manufacture.

A small fraction of the wave-associated energy generated by the rotating load or impulsive load of machine tools, transmitted to the human hand-arm system has the energy to produce Osteoarthritis, Cartilage damage, Microvascular complications and nervous system disorders. Since 19th April 1993, prescription concerns for carpal tunnel syndrome. Carpal tunnel syndrome is a prescribed disease only for the use of handheld vibrating tools. It is unclear whether the disorder is a consequence of the vibration or the posture and grip required to use such tools. Other factors associated with carpal tunnel syndrome are various hormonal non-occupational factors including female sex, pregnancy, oral contraceptive use, bilateral oophorectomy, diabetes mellitus, and rheumatoid arthritis.

Prescription for the hand-arm vibration syndrome is updated. In 2004, the Industrial Injury Advisory Council recommended that prescription should be extended to include the sensorineural component:

1. persistent numbness or persistent tingling, or both, together with

2. significant and measurable reduction in both sensory perception and manual dexterity

Recommendation of the IIAC-July 2006 concerns prescription for the hand-arm vibration syndrome. Carpal tunnel syndrome is recommended by the Industrial Injuries Advisory Council. In addition, the are prescription for two types of job:

1. The use, at the time the symptoms first develop, of handheld powered tools whose internal parts vibrate to transmit the vibration to the hand, but excluding those which are solely powered by hand; or

2. Repeated palmar flexion and dorsiflexion of the wrist for at least 20 hours per week in those who have undertaken such work for at least 12 months in aggregate in the 24 months before the onset of symptoms.

\section{Conclusion}

The peculiar aspects of this research concern the analysis of postures of the human hand-arm system and predisposing factors of occupational hand-transmitted vibration exposures. The axis with the greatest vibratory effects on the hand-arm position depends on the machine tool. The rotation speed of the machine tool is the mechanical quantity most correlated 
to the peak-to-peak and peak values of the vibratory signals acquire on the human hand-arm system. The flexion angle of maximum efficiency is between $80^{\circ}$ and $90^{\circ}$ for the biceps and the brachioradialis between $100^{\circ}$ and $110^{\circ}$. This research has not developed any finite element models of the entire hand-arm system. There is a need to propose a complex finite element model to estimate biodynamic responses for the substructures of the hand-arm system.

\section{List of Symbols}

$\begin{array}{cl}\text { Adjusted } \mathrm{R}^{2} & \text { adjusted R-squared } \\ \mathrm{C} & \begin{array}{l}\text { centrifugal component } \\ C_{1} C_{2} C_{3} C_{4}\end{array} \\ \mathrm{D} & \text { constants of integration } \\ \text { diameter of the handle } \\ \mathrm{E} & \text { Young's modulus } \\ \mathrm{I} & \text { moment of inertia of the beam RPM } \\ \mathrm{L} & \text { lenght } \\ \mathrm{P} & \text { power } \\ \mathrm{Q} & \text { generalized force } \\ \mathrm{R}-\mathrm{squared} & \text { coefficient of determination } \\ \mathrm{RPM} & \text { number of revolutions per min } \\ \mathrm{RMS} & \text { root mean square } \\ \mathrm{RMSE} & \text { root mean squared error } \\ \mathrm{S} & \text { cross-sectional area } \\ \mathrm{T} & \text { normal (tangential) component } \\ \mathrm{w} & \text { response of hand arm system } \\ \omega_{n} & \text { natural frequency } \\ \mathrm{x}, \mathrm{y} \mathrm{z} & \text { axis }\end{array}$

\section{REFERENCES}

[1] Dong, R.G., Welcome, D.E., Xueyan, S. Xu, McDowell, T.W., Chen, Q., Modeling Analyses of the Vibration Response Characteristics of a Handheld Workpiece in Grinding Process, 14th International Conference on Hand-Arm Vibration, 21-24 May 2019, 73-74.

[2] Fattorini, L., Tirabasso, A., Lunghi, A., Di Giovanni, R., Sacco, F., Marchetti, E., Muscular synchronization and hand-arm fatigue, International Journal of Industrial Ergonomics, 62, 2017, 13-16.

[3] Sisto, R., Botti, T., Cerini, L.,Di Giovanni, R., Marchetti, E., Lunghi, A., Sacco, F., Sanjust, F., Tirabasso, A.,
Moleti, A., Synergistic effects of noise and hand-arm vibration on distorsion product otoacoustic emission in healthy subjects, International Journal of Industrial Ergonomics, 62, 2017, 48-54.

[4] Marchetti E., Sisto R., Lunghi A., Sacco F., Sanjust F., Di Giovanni R., Botti T., Morgia F., Tirabasso A., An investigation on the vibration transmissibility of the human elbow subjected to hand-transmitted vibration, Int. J. Ind. Ergon. 62 (2017), 82-89.

[5] Moschioni, G., Saggin, B., Tarabini, M., Prediction of data variability in hand-arm vibration measurements, Measurement 44(9):1679-1690, 2011, DOI: 10.1016/j.measurement.2011.06.022.

[6] Cavacece M., Aghilone G., Intra-subject Variability of Impact Shock of Foot on the Ground during Walking and Running Activities, Universal Journal of Public Health, 2018,6 (4), 167-172, 167-172.DOI: 10.13189/ujph.2018.060401

[7] Cavacece, M., Aghilone, Biodynamic responses at the fingers and at the palm of the human hand-arm system under different vibration source. 14th International Conference on Hand-Arm Vibration, 21-24 May 2019, 77-78.

[8] Cavacece, M., Aghilone, G., Strategies for occupational safety and health to prevent the effects of the exposure to hand-arm vibration. 14th International Conference on Hand-Arm Vibration, 21-24 May 2019, 131-132.

[9] Cavacece, M., Figliolini, G., Lanni, C., Vertical Vibrations of the Vehicle Excited by Ride Test. In: Rao Y.V.D., Amarnath C., Regalla S.P., Javed A., Singh K.K. (eds) Advances in Industrial Machines and Mechanisms. Lecture Notes in Mechanical Engineering. Springer, Singapore, 2021, 631-642, https://doi.org/10.1007/978981-16-1769-0-57.

[10] Griffin M., Handbook of Human Vibration, Academic Press, 1996, ISBN: 9780123030412. 